\title{
Ponderomotive barrier for plasma particles on the boundary of astrophysical jets
}

\author{
Anna A. Dubinova ${ }^{1}$ and Vladimir V. Kocharovsky ${ }^{1}$ \\ ${ }^{1}$ Institute of Applied Physics of the Russian Academy of Sciences, Nizhny Novgorod, Russia \\ email: anndub@gmail.com, kochar@appl.sci-nnov.ru
}

\begin{abstract}
We study kinetics of plasma particles on internal inhomogeneities and on the boundary of astrophysical jets in the presence of intensive low-frequency electromagnetic (surface and bulk waves). These waves with energy density exceeding or of the same order as the particle kinetic energy density can be generated due to the non-equilibrium state of plasma and lead to the jet stratification at various scales. The main reason is a ponderomotive force which is able to change dramatically the particle behavior, the plasma density cross-section profile and the wave collimation. We present results obtained on the basis of our simple ponderomotive model of the self-consistent analysis of the electromagnetic wave propagation and the formation of the plasma density profile.
\end{abstract}

Keywords. galaxies: jets, plasmas, waves.

\section{Introduction}

We describe the structure of Poynting-dominated jets (Blandford R., 2003, Guthmann A. W., 2002) in which particle collimation and boundary formation are strongly influenced by low-frequency electromagnetic waves propagating in the jet body and along the boundaries of the jet plasma waveguide. Originated from the guided waves the gradient ponderomotive force might be responsible for the formation of self-consistent plasma density profile $\rho=m n$. We expect the role of the ponderomotive force to be as important as the role of plasma pressure, $p$, inhomogeneous particle motion with bulk velocity, $u$, and quasistatic magnetic field pressure, $B_{0}^{2} / 8 \pi$. Previously, the ponderomotive effects were usually discussed with respect to particle acceleration along jets or with respect to the bulk velocity profile formation (e.g. Lundin R. et al., 2006).

This can be explained by means of the generalized Bernoulli law with a corresponding term which stands for the ponderomotive potential $w_{\text {pond }}$

$$
\frac{\rho u^{2}}{2}+p+\frac{B_{0}^{2}}{8 \pi}+w_{\text {pond }} \cong \text { const. }
$$

At large scales (of order of the jet radius $R_{\text {jet }}$ ) the MHD approach is usually applicable (e.g. Komissarov S. S., 2010 et al., and McKinney J.C. et al., 2009). At small scales up to the plasma skin depth and the electromagnetic wavelength the kinetic approach is inevitable to describe, for example, the steepening of jet boundaries and inhomogeneities inside jets. Generally speaking, we should take into consideration also wave and plasma turbulence (e.g. Sagdeev R. Z., 1979; Litvak A. G., 1986) but we restrict ourselves to studying steady-state configurations Fig. 1. 


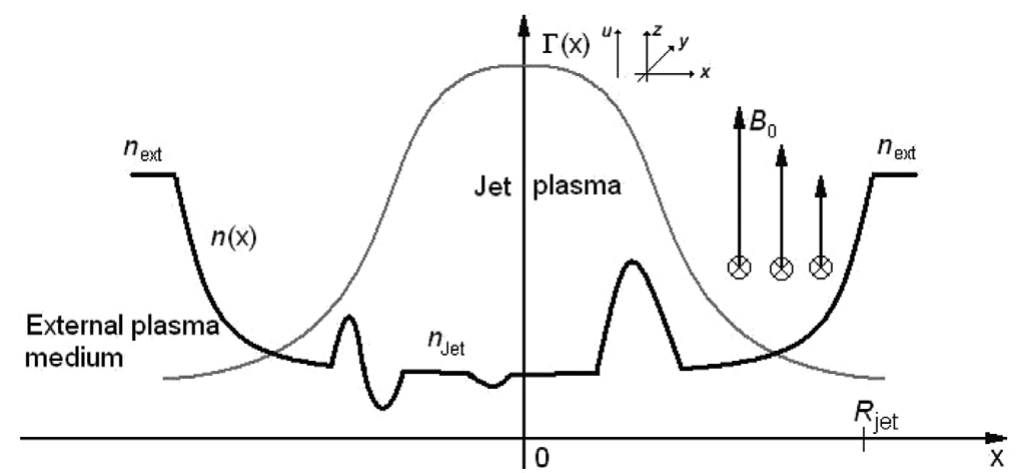

Figure 1. Scheme of the jet cross-section profile $(n(x)$ is the plasma density inside the jet and $n_{\text {ext }}$ the plasma density outside the jet, $\Gamma(x)$ the bulk Lorents factor).

\section{Features of ponderomotive forces}

The well-known non-relativistic expression for the gradient ponderomotive (Miller) force in the presence of a plane monochromatic wave $\sim \boldsymbol{E}(\boldsymbol{r}) e^{i \omega t}$ at frequency $\omega$ reads (Lundin R. et al., 2006)

$$
\boldsymbol{F}_{\mathrm{pe}}\left(\boldsymbol{r}_{\mathrm{d}}\right)=-\nabla \Phi_{\mathrm{pe}}, \quad \Phi_{\mathrm{pe}}\left(\boldsymbol{r}_{\mathrm{d}}\right)=\frac{e^{2}}{4 m \omega^{2}}\left|\boldsymbol{E}\left(\boldsymbol{r}_{\mathrm{d}}\right)\right|^{2} ; \quad \frac{m\left|\dot{\boldsymbol{r}}_{\mathrm{d}}\right|^{2}}{2}+\Phi_{\mathrm{pe}}\left(\boldsymbol{r}_{\mathrm{d}}\right)=\text { const },
$$

where $e$ and $m$ are charge and mass of a plasma particle (electron), $\boldsymbol{r}_{\mathrm{d}}$ is radius-vector of a particle position averaged over the wave period $2 \pi / \omega$. Taking into consideration the effects caused by an external quasistatic magnetic field, $B_{0}$, and a finite rate of particle collisions (or turbulence scattering), $\nu$, one arrives at the more general expression for the ponderomotive force

$$
\begin{aligned}
\boldsymbol{F}_{\mathrm{pe}}\left(\boldsymbol{r}_{\mathrm{d}}\right)= & -\frac{e^{2}}{4 m \omega^{2}}\left(\frac{1}{1+\nu^{2} / \omega^{2}} \nabla\left|\boldsymbol{E}_{\|}\right|^{2}+\frac{1+\omega_{\mathrm{B}} / \omega}{\left(1+\omega_{\mathrm{B}} / \omega\right)^{2}+\nu^{2} / \omega^{2}} \nabla\left|\boldsymbol{E}_{\perp}^{+}\right|^{2}\right. \\
& +\frac{1-\omega_{\mathrm{B}} / \omega}{\left(1-\omega_{\mathrm{B}} / \omega\right)^{2}+\nu^{2} / \omega^{2}} \nabla\left|\boldsymbol{E}_{\perp}^{-}\right|^{2}+\frac{2 \nu / \omega}{1+\nu^{2} / \omega^{2}}\left|\boldsymbol{E}_{\|}\right|^{2} \nabla \Psi_{\|} \\
& \left.+\frac{2 \nu / \omega}{\left(1+\omega_{\mathrm{B}} / \omega\right)^{2}+\nu^{2} / \omega^{2}}\left|\boldsymbol{E}_{\perp}^{+}\right|^{2} \nabla \Psi_{\perp}^{+}+\frac{2 \nu / \omega}{\left(1-\omega_{\mathrm{B}} / \omega\right)^{2}+\nu^{2} / \omega^{2}}\left|\boldsymbol{E}_{\perp}^{-}\right|^{2} \nabla \Psi_{\perp}^{-}\right),
\end{aligned}
$$

where $\Psi_{\|}, \Psi_{\perp}^{+}, \Psi_{\perp}^{-}$are phases of the corresponding electric field components - longitudinal and transverse circular-polarized ones (with respect to $\boldsymbol{B}_{0}$ ), $\omega_{\mathrm{B}}=e B_{0} / m c$ is the cyclotron frequency (Litvak A. G., 1986). A cyclotron resonance at $\omega=\omega_{\mathrm{B}}$ results in strong dependence of the ponderomotive force on the effects of particle collisions and particle scattering. These effects is also crucial for the dynamics of waves at frequencies at the vicinity of the cyclotron frequency as well as the plasma frequency. In the general case of non-monochromatic electromagnetic field contributions of all the harmonics should be summed incoherently. For a continuous spectrum of incoherent waves it is possible to replace summing by integrating.

We have analyzed this force and the importance of the effective ponderomotive potential for various types of low-frequency waves and their simple possible spectra and found out that there is a wide range of wave and plasma parameters for which the ponderomotive separation of rarefied an dense plasma layers could exist at relatively small scales. Roughly speaking, it happens when the ponderomotive barrier exceeds the average particle energy. That requires the wave energy density of the order of the equipartition value. 

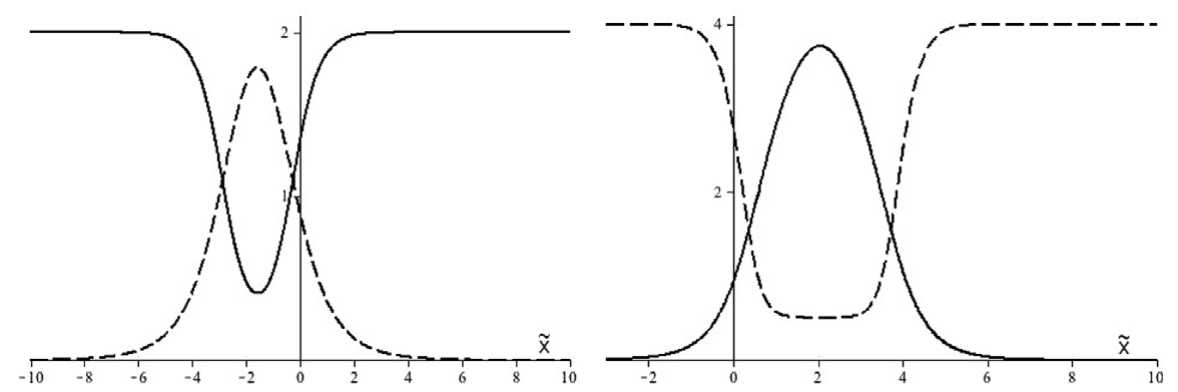

Figure 2. Typical self-consistent solutions of the system (3.2): $\tilde{\omega}_{\mathrm{p}}^{2}(\tilde{x})$ (solid line) and $\tilde{E}(\tilde{x})$ (dashed line) at $\tilde{k}_{z}^{2}=0, \kappa_{\infty}^{2}=1$ (the left graph) and at $\tilde{k}_{z}^{2}=0.5, \kappa_{\infty}^{2}=3$ (the right graph).

\section{A simple model}

For illustration, let us consider a $s$-polarized monochromatic wave incident on the onedimensional inhomogeneous plasma with the density profile determined by the pressure balance. The net pressure at certain point $x$ is a sum of the thermal plasma pressure and the ponderomotive pressure created by the incident electromagnetic wave. We are eager to obtain a stationary self-consistent structure of the electromagnetic field and the plasma density. Combining the Helmholtz equation for a real amplitude of the electric field $y$-component and the pressure balance equation we arrive at the pair of normalized self-consistent equations with boundary conditions

$$
\begin{aligned}
\frac{\partial \kappa^{2}}{\partial \tilde{x}}+\left(\kappa^{2}+1-\tilde{k}_{\mathrm{z}}^{2}\right) \tilde{E} \frac{\partial \tilde{E}}{\partial \tilde{x}}=0, & \frac{\partial^{2} \tilde{E}}{\partial \tilde{x}^{2}}-\kappa^{2} \tilde{E}=0, \\
\kappa^{2}(x \rightarrow+\infty)=\kappa_{\infty}^{2}, \quad \tilde{E}(x \rightarrow+\infty)=0, & \frac{\partial \tilde{E}}{\partial \tilde{x}}(x \rightarrow+\infty)=0,
\end{aligned}
$$

where we ignore the magnetic field and introduce the following denotations

$$
\tilde{E}=\frac{E e}{\omega \sqrt{2 m k_{\mathrm{B}} T}}, \quad \tilde{\omega}_{\mathrm{p}}^{2}=\frac{\omega_{\mathrm{p}}^{2}}{\omega^{2}}, \quad \tilde{x}=\frac{x \omega}{c}, \quad \tilde{k}_{\mathrm{z}}^{2}=\frac{k_{\mathrm{z}}^{2} c^{2}}{\omega^{2}}, \quad \kappa^{2}=\tilde{\omega}_{\mathrm{p}}^{2}-1+\tilde{k}_{\mathrm{z}}^{2} .
$$

Here $\omega_{\mathrm{p}}$ is the local plasma frequency proportional to the plasma density, $k_{\mathrm{z}}$ the wave number along $z$ axis, $T$ the plasma temperature which is assumed constant, $c$ the speed of light in vacuum and $k_{\mathrm{B}}$ the Boltzman constant.

System (3.2) can be easily reduced to the non-linear Schödinger equation

$$
\partial^{2} \tilde{E} / \partial \tilde{x}^{2}-\left[\left(\kappa_{\infty}^{2}+1-\tilde{k}_{\mathrm{z}}^{2}\right) \exp \left(-\tilde{E}^{2}\right)-\left(1-\tilde{k}_{\mathrm{z}}^{2}\right)\right] \tilde{E}=0
$$

It gives soliton-like solutions which can be found numerically. They are demonstrated on the graphs on Fig. 2. It is clear to see that the wave field is localized and well-collimated and that the jet boundary width may be of the order of the electromagnetic wavelength. The higher the external plasma frequency and the larger longitudinal wavelength $k_{z}$ the deeper and wider the plasma channel is.

\section{Problems and discussions}

Similar self-consistent $1 \mathrm{D}$ analysis for $p$-polarized waves (both bulk and surface) is more complicated because of the presence of the electric field component along the plasma density gradient. This fact leads to the resonant growth of the electric field near the wave reflection point and the resonant excitation of strong plasma waves at the vicinity 
of the point where the dielectric permittivity is equal to zero. However, these effects do not change the general conclusion that the small-scale plasma stratification and the formation of the sharp boundary could be typical for the Poynting-dominated jets due to the ponderomotive influence of low-frequency electromagnetic waves in the process of their self-channeling. There are also a number of qualitative arguments indicating that scattering, dissipation and various wave instabilities could affect the bulk acceleration, the effective particle temperature, the structure of boundary layers, the intrajet turbulence, the anomalous plasma conductivity, the magnetic field diffusion, the evolution of jet opening angle, etc. The rigorous analysis of all these problems requires additional (usually unavailable) information on jets including spectra of low-frequency powerful waves, their instability rates and scales, features of nonlinear stabilization and slow evolution of smallscale plasma turbulence, and so on.

\section{Conclusions}

In the presence of intensive electromagnetic surface and bulk waves as well as intensive plasma waves the gradient ponderomotive force can have strong influence on the kinetics of weakly collisional plasma particles and as a consequence on the plasma stratification inside jets and on the boundary plasma layer between the jet and the external medium. The electromagnetic waves are able to create a channel which they cannot escape if their frequency is below the plasma frequency of the external medium.

We point out the vital importance of the gradient ponderomotive force for the formation of internal inhomogeneities and the boundary of Poynting-dominated astrophysical jets. Our preliminary results make it clear that further development of the ponderomotive model of the jet boundary confinement is essential for understanding the processes of jet formation and collimation. The present work is the first step on the way to create a self-consistent ponderomotive model describing the collimation of astrophysical jets along with due analysis of the self-channeling of the low-frequency electromagnetic waves. There is no doubt that the advanced study of the problem will require numerical simulations which combine large-scale MHD and small-scale kinetic approaches.

\section{References}

Litvak, A. G. 1986, Review of Plasma Physics, 10, 293

Sagdeev, R. Z. 1979, Rev. Mod. Phys., 51, 1

McKinney, J. C. \& Bladford, R. D. 2009, Mon. Not. R. Astron. Soc., 394, L126

Guthmann, A. W., Georganopoulos, M. et al. 2002, Relativistic flows in astrophysics, Springer

Komissarov, S. S., Vlahakis, N. \& Königl A. 2010, Mon. Not. R. Astron. Soc., 104, 17

Blandford, R. 2003, ASP Conference series, 290, 267

Lundin, R. \& Guglielmi, A. 2006, Space Sci. Rev., 127, 1 